\title{
Erratum to: Emergency of Ocular Adnexa
}

\author{
Haoyu Chen, Dan Chao, Guihua Xu, Chen Lin, \\ and Zeyi Li
}

\section{Erratum to:}

Chapter 10 in: H. Yan (ed.), Ocular Emergency, Ocular Trauma, https://doi.org/10.1007/978-981-10-6802-7_10

The Original Volume was inadvertently published without including the figures in Chapter 10. The figures have been included in this updated Volume. 